\title{
Nutrição e envelhecimento: algumas reflexões sobre a integração entre ensino, pesquisa e extensão no Projeto Nutrição e Terceira Idade
}

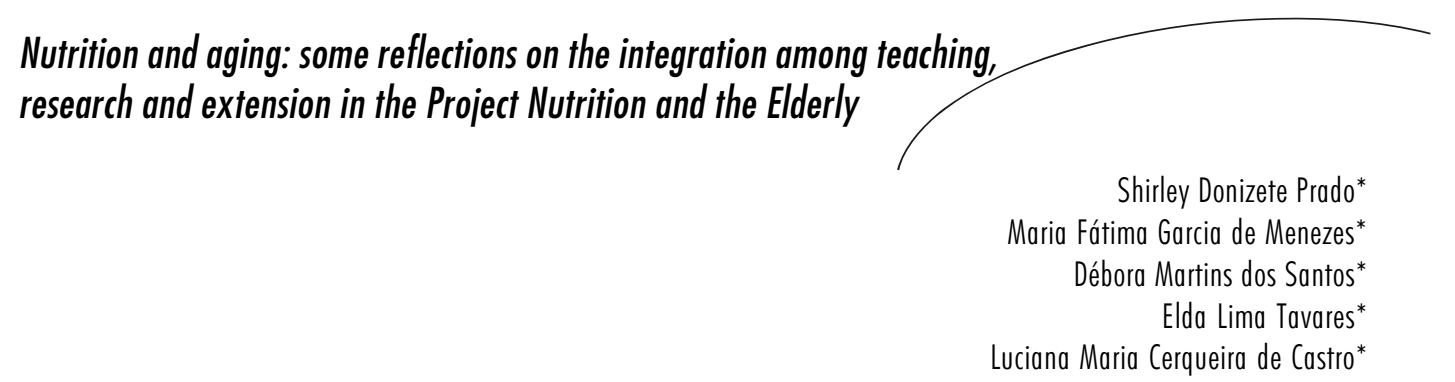

Resumo

Buscamos discutir a articulação entre pesquisa e extensão no âmbito do Projeto de Extensão Nutrição e Terceira Idade desenvolvido pelo Instituto de Nutrição da Universidade do Estado do Rio de Janeiro no qual implementamos um conjunto de atividades individuais e coletivas organizadas em quatro eixos principais: (a) assistência nutricional individual e personalizada; (b) avaliação nutricional individual: análise antropométrica e dietética; (c) pesquisa sobre comportamento alimentar: cultura, envelhecimento e saúde; (d) práticas educativas: desenvolvimento de metodologias. Para tanto, tomamos o pensamento de Nogueira sobre a extensão como uma relação de mão dupla entre Universidade e Sociedade que possibilita a oportunidade da elaboração da práxis de um conhecimento acadêmico; os conceitos de ciência e de técnica em Canguilhem a partir de suas discussões sobre a Medicina e o campo da saúde; e os referenciais teóricos de Foucault relativos a saberes em seus debates acerca do campo das Ciências Humanas. Identificamos algumas limitações importantes a exigirem de nós novas posturas. Principalmente, a necessidade de escrever, de registrar o que fazemos. Estamos gerando novos conhecimentos e saberes, novas técnicas que estão ainda encerradas em nossos muros. Múltiplos fatores podem ser elencados na identificação da determinação desses limites. Certamente, ações individuais e institucionais precisam ser implementadas para a superação desses limitantes que, acreditamos, sejam característicos, em algum grau, das atividades extensionistas na UERJ e talvez no Brasil.

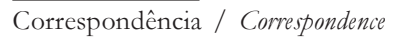

Shirley Donizete Prado

* Departamento de Nutrição Social do Instituto de Nutrição da Universidade do Estado do Rio de Janeiro

Rua São Francisco Xavier, 524, $12^{\circ}$ andar, Bloco D, sala 12.02820559-900 Rio de Janeiro, RJ - Brasil

\author{
Palavras-chave: \\ programas de \\ nutrição; envelheci- \\ mento; ensino; \\ pesquisa aplicada; \\ assistência \\ ambulatorial
}




\section{Abstract}

We try to discuss the articulation between research and extension within the extension project "Nutrition and the Elderly", carried out by the Nutrition Institute at Rio de Janeiro State University (UERJ), which develops several individual and collective activities organized in four main branches: (a) individual and personalized nutritional aid; (b) individual nutritional evaluation; (c) research about feeding behavior: culture, aging and health; (d) educational practices: development of methodologies. For this purpose, we take Nogueira's ${ }^{11}$ ideas on extension as a two-way relation between university and society which allows the elaboration of a praxis on academic knowledge; Canguilhem's concepts on science and technique, based on his discussions on Medicine and the Health area; and Foucault's theoretical references concerning knowledge and his debates on the Human Sciences. We identified some important limitations that require new viewpoints, mainly the need for taking note, recording all we do. We create new knowledge, new techniques that are still unveiled. Many factors determine these limits. Individual and institutional actions must be implemented to overcome these limits that, in our opinion, characterize to some degree extension activities at UERJ and maybe in Brazil.
Key words: nutrition programmes; aging; teaching; applied research; ambulatory care

\section{Integração entre ensino, pesquisa e extensão: referenciais teóricos.}

O papel da Universidade relaciona-se, tradicionalmente, à produção, reprodução e transmissão de conhecimento. Mais recentemente, as atividades de extensão vêm ganhando espaços expressivos no cenário universitário como mais uma oportunidade de ensinar e de aprender. A Universidade do Estado do Rio de Janeiro (UERJ), é uma das mais fortes expressões nacionais na implementação de atividades extensionistas; no ano de 2006, há na Sub-Reitoria de Extensão e Cultura 138 projetos cadastrados, somente na área estratégica da Saúde ${ }^{21}$.

Desse sub-conjunto faz parte o Projeto Nutrição e Terceira Idade (PNTI). Criado em 1993, corresponde a parceria entre o Depar- tamento de Nutrição Social do Instituto de Nutrição da Universidade do Estado do Rio de Janeiro (UERJ) e a Universidade Aberta da Terceira Idade (UnATI), que é um Centro Colaborador da Organização Mundial da Saúde estruturado como núcleo de formação de recursos humanos, estudos, debates, centro de convivência e de assistência à população idosa na UERJ.

No Projeto Nutrição e Terceira Idade, desde sua criação, são desenvolvidas diferentes atividades com indivíduos com 60 anos e mais. Os usuários apresentam perfil sóciodemográfico semelhante ao da população idosa do Município do Rio de Janeiro ${ }^{6}$. Em sua maioria são mulheres, aposentadas e/ ou pensionistas, viúvas, solteiras e descasadas. A escolaridade é superior a média nacional, com grande parte apresentando segundo grau completo ${ }^{17}$. 
A trajetória do PNTI traz as marcas de parceiros que foram conquistados e que, em muito enriqueceram o trabalho: alunos do Curso de Graduação em Nutrição, bolsistas, estagiários, voluntários, residentes de diversas áreas, profissionais e docentes oriundos de várias unidades acadêmicas e de outras instituições e idosos participantes das atividades.

No PNTI são realizadas ações de ensino para alunos de graduação em Nutrição: um espaço importante na qualificação de futuros profissionais através de aulas práticas de diferentes disciplinas e campo de estágio da área de Nutrição em Saúde Coletiva. No espaço da pesquisa, o Projeto vem possibilitando a realização de diferentes estudos e gerando conhecimentos e saberes específicos acerca da população idosa que participa das atividades aí oferecidas. No que se refere à extensão, são oferecidos serviços e assistência em nutrição - consultas individuais e personalizadas, cursos, grupos de reflexão e promoção de eventos. O presente trabalho tem por objetivo discutir algumas questões pertinentes à articulação das ações de extensão e pesquisa no âmbito do Projeto de Nutrição e Terceira Idade, buscando identificar limites e possibilidades. Para tanto, são considerados alguns balizamentos conceituais apresentados a seguir.

Historicamente, a extensão tem início a partir de duas vertentes bem diferenciadas. $\mathrm{O}$ modelo europeu que entendia a extensão como um espaço através do qual seriam disseminados conhecimentos técnicos à população carente; uma forma que a universidade teria para se redimir do seu distanciamento de um determinado grupo que não teria aces- so a ela; na Universidade Livre de São Paulo, essa linha de atuação é caracterizada pela realização de conferências semanais gratuitas dirigidas a população. $\mathrm{O}$ modelo americano coloca na extensão a perspectiva de prestação de serviços, de certa forma reduzindo o papel do Estado para com suas próprias obrigações. Em Minas Gerais, a partir dessa referência, tem início a extensão por meio de prestação de assessoria a pequenos agricultores. Como afirma Castro3: "O que se nota é que, além do caráter opcional, a extensão aparece como a maneira de aproximar a Universidade da população, principalmente de forma assistencial, esvaziada de qualquer significado emancipatório, mas como uma forma de realização das políticas de governo." (p. 25)

A extensão inserida na estrutura universitária vem buscando, ao longo de sua trajetória, romper com diferentes rótulos que a ela se associavam (ou que permanecem até os dias de hoje), como: invasão cultural, assistencialismo, messianismo, prestação de serviços. A partir de eventos e processos marcantes como a criação do Fórum Nacional de Pró-Reitores de Extensão das Universidades Públicas, entre outros, novos rumos foram dados à extensão a partir da década de 1980. Reconhece-se como imprescindível o contato de setores da universidade com os serviços de saúde, de educação, de assistência social, entre outros, como caminhos para a construção de uma maior articulação entre a academia e a vida cotidiana ${ }^{16}$. Não se trata de mera divulgação de informações ao público, mas como definição no referido Fórum ${ }^{11}$, de um "...processo educativo, cultural e científico que articula o ensino e a pesquisa de forma indissociável e viabiliza a relação transformadora entre Universida- 
de e a sociedade. A extensão é uma via de mão dupla, com trânsito assegurado à comunidade acadêmica, que encontrará, na sociedade, a oportunidade da elaboração da práxis de um conhecimento acadêmico. No retorno à Universidade, docentes e discentes trarão um aprendizado que, submetido à reflexão teórica, será acrescido àquele conhecimento. Este fluxo, que estabelece a troca de saberes sistematizados-acadêmico e popular, terá como conseqüência: a produção de conhecimento resultante do confronto com a realidade brasileira e regional; e a democratização do conbecimento e a participação efetiva da comunidade na atuação da Universidade. Além de instrumentalizadora desse processo dialético de teoria/prática, a extensão é um trabalho interdisciplinar que favorece a ação integrada do social..." (p.11).

Assim, a extensão pressupõe a relação entre Universidade e Sociedade, que os pode irmanar em uma ação solidária, entre sujeitos ativos, autônomos e responsáveis; sujeitos de conhecimento, que refletem, modificam seus conhecimentos e buscam caminhos para a construção/reconstrução do mundo ${ }^{1}$.

Em comparação com a extensão, a ciência ocupa, há muito, um lugar privilegiado no mundo moderno, especialmente, nos meios acadêmicos; seu desenvolvimento, em particular no que se refere aos aspectos tecnológicos, colocam-na em posição de destaque por conta do estatuto de verdade que confere às conclusões sobre os mais diversos fenômenos que aborda ${ }^{19}$.

A identificação daquilo que se constitui $c i$ ência encontra fundamentos na Epistemologia de Bachelard através de suas análises acerca da cientificidade nos procedimentos de pesquisa no campo das "ciências da natureza ou da matéria": a Física e a Química ${ }^{7}$. Partindo dessas mesmas referências Canguilhem ${ }^{2}$ volta-se para as "ciências da vida" - Biologia e Fisiologia - distinguindo-as da Medicina, clínica e terapêutica, construída a partir do sofrimento, da doença de um ser, de obstáculos patológicos, entendendo-a como situada em um outro campo que não o da ciência, mas o das técnicas de alívio do sofrimento, da cura, um campo de subjetividades, de valores, de construção incessante de novas normalidades. Operando um certo deslocamento em relação à Epistemologia, encontramos em Michel Foucault ${ }^{4}$ a Arqueologia do Saber, cujas análises estão centradas na questão do homem, na constituição histórica das "ciências do homem" na modernidade: uma nova região ao lado dos campos "da natureza" e "da vida"; trata-se de uma nova abordagem analítica que guarda em comum com a Epistemologia a exigência de realizar uma análise conceitual profunda e que tem a Filosofia e a História como elementos chave para seu desenvolvimento, mas que se distingue pelo abandono à questão da cientificidade, cerne do projeto epistemológico. As palavras sintéticas de Machado $^{7}$ são esclarecedoras em relação a essas distinções: "Parece-nos mesmo que a riqueza do método arqueológico é ser um instrumento capaz de refletir sobre as ciências do homem enquanto saberesinvestigando suas condicões de existência através da análise do que dizem, como dizem e porque dizemneutralizando a questão da cientificidade e escapando assim ao desafio impossivel da recorrência, sem, no entanto, abandonar a exigência de realizar uma análise conceitual capaz de estabelecer descontinuidades, não certamente epistemológicas, mas arqueológicas, isto é, situadas ao nível dos saberes." (p. 11). 
Essencialmente, o trabalho de Foucault ${ }^{4}$ implica um deslocamento metodológico da Epistemologia para a Arqueologia que coloca, em lugar do conhecimento, um novo objeto: o saber. Enquanto que as análises epistemológicas não são aplicáveis para formações discursivas não científicas, a Arqueologia é tomada para dar conta de disciplinas (formações discursivas) não propriamente científicas, o que se dá por meio da análise da positividade do que foi efetivamente dito. Enquanto que a positividade na epistemologia mantém correspondência com a cientificidade, na arqueologia ela é capaz de caracterizar e individualizar um discurso como saber, como portador de verdade.

Partindo dos referenciais epistemológicos, podemos pensar na extensão como um investimento situado num espaço que pode articular campos científicos e técnicas de intervenção - como em Canguilhem ${ }^{2}$, a Biologia e a Fisiologia aproximam-se da Medicina; a Fisiologia da Nutrição aproxima-se dos nutricionistas em seus consultórios diante de patologias mais próprias de pacientes idosos; a Epidemiologia fundamentando a definição de políticas e programas de atenção à saúde e nutrição de populações idosas. Ou, se são tomadas premissas de natureza arqueológicas, torna-se possível visualizar a extensão como iniciativas que podem relacionar a produção de saberes e as técnicas de intervenção - a Sociologia, a Antropologia em suas relações com as práticas educativas em alimentação e nutrição no processo de envelhecimento.

No que se refere ao ensino, a pesquisa e a extensão aparecem como elementos valori- zados nas Diretrizes Curriculares Nacionais dos Cursos de Graduação em Enfermagem, Medicina e Nutrição emanadas pelo Ministério da Educação ${ }^{10}$. Consta de seus princípios "Fortalecer a articulação da teoria com a prática, valorizando a pesquisa individual e coletiva, assim como os estágios e a participação em atividades de extensão"

A pesquisa insere-se nas diretrizes curriculares, como uma prerrogativa fundamental para a formação profissional que possibilita o desenvolvimento, na graduação, de um olhar científico, curioso, indagador e criativo ${ }^{5}$. Segundo Maldonado ${ }^{8}$ “.... a participação em atividades de pesquisa estimula a criatividade e curiosidade e incrementa a atitude crítica dos alunos, que desenvolvem uma nova postura em relação ao saber e se tornam mais motivados para aprender."(p.155).

O mesmo pode ser dito em relação à extensão. Essas referências conceituais vêm orientando as atividades no PNTI no sentido da proposição e implementação de um conjunto de ações que possibilitam um certo trânsito entre distintas atividades que correspondem, ao menos em parte, em concretizações de articulação entre ensino, pesquisa e extensão. Algumas reflexões sobre limites e possibilidades dessas iniciativas encontram-se apresentadas a seguir.

\section{Integração entre ensino, pesquisa e extensão no âmbito do Projeto Nutrição e Terceira Idade: limites e possibilidades}

O envelhecimento da população brasileira trouxe para a Universidade desafios no sentido de buscar respostas para questões relati- 
vas à identificação de necessidades, à compreensão dos processos de saúde-doença, ao cuidado individual e à intervenção em face de demandas específicas, entre outras. Como afirma Veras ${ }^{23}$, é necessário “...mudar conceitos já enraizados e utilizar novas tecnologias, com inovação e sabedoria, a fim de alcançar de forma justa e democrática a eqüidade na distribuição dos serviços e facilidades para o grupo populacional que mais cresce em nosso país." (p.700)

Nesse cenário, as atividades do Projeto Nutrição e Terceira Idade tiveram início buscando a construção de articulações entre ensino, pesquisa e extensão. Essas proposições foram, aos poucos, se transformando, buscando uma postura cada vez mais reflexiva diante da realidade dinâmica, complexa, com múltiplos determinantes, que colocava sempre a necessidade de maior refinamento de instrumental para conhecer, compreender e agir. As dúvidas, incertezas, erros, acertos, impuseram reflexões, transformando conhecimentos, saberes e práticas. Melo et al nos dizem que "... a idéia chave é a de que a inserção na realidade, a interação entre todos os atores envolvidos e o enfrentamento responsável dos problemas detectados proporcionam uma prática coletiva onde o agir, o aprender e o pesquisar se somam, se complementam, se potencializam, produzindo dessa forma um fecundo processo de formação e transformação de sujeitos, de saberes e de realidades sociais objetivas." (p.1)

Por esses caminhos, foi definido o conjunto de ações implementados nos dias atuais e que pode ser apresentado por meio de quatro eixos principais: (a) assistência nutricional individual e personalizada; (b) estudos sobre avaliação nutricional individual: análise antropométrica e dietética; (c) estudos sobre comportamento alimentar: cultura, envelhecimento e saúde; (d) práticas educativas: desenvolvimento de metodologias.

No plano da assistência nutricional individuale personalizada, a promoção da saúde de idosos é a marca fundamental do Projeto. No interior do Núcleo de Atenção ao Idoso da UnATI, recebemos usuários com vistas a proceder avaliação nutricional individual e orientação dietética personalizada de caráter preventivo em face dos diagnósticos estabelecidos. Essa atividade é coordenada pelo corpo docente e da qual participam alunos do Curso de Graduação em Nutrição (bolsistas de extensão, estagiários e voluntários). É o espaço que mais se aproxima das técnicas de diagnóstico clínico, da arte da cura e do alívio das dores e desconfortos, se olharmos como Canguilhem $^{2}$ vê a Medicina. Potencialmente, este corresponde ao lócus de geração de publicações sobre modelos de atenção nutricional especificamente voltado para idosos.

Para além da assistência articulada à formação profissional, é a partir daí que são realizados estudos no âmbito da avaliação nutricional individual: análises antropométrica e dietética da população atendida; esses estudos acontecem dentro de uma perspectiva mais ampla na qual também são analisadas múltiplas dimensões da vida do idoso em conjunto com outros profissionais que correspondem aos parceiros institucionais. No momento do atendimento nutricional individual são levantados dados qualitativos e quantitativos sobre a situação nutricional e alimentar dos idosos. Esses dados que servem tanto para a condução da 
orientação nutricional individualizada como para a produção de conhecimento sobre perfil dietético e antropométrico da população atendida incluindo associação entre dieta, condições de saúde e de vida. Aqui é desenvolvida a produção de conhecimento tal como a encontramos em Canguilhem ${ }^{2}$ ao tratar da Fisiologia e da Biologia: o lugar da cientificidade partilhada com alunos de Graduação, cujos resultados contribuem para o embasamento da construção das práticas ambulatoriais.

Um outro eixo de estudos volta-se para o comportamento alimentar: cultura, envelhecimento e saúde. Conduzido por docentes com a presença de graduandos em Nutrição, materializa-se através de entrevistas semi-estruturadas e das Oficinas de Produção de Textos, nas quais idosos com interesse pela ficção, pelas narrativas ou outras formas de expressão escrita, revelam suas experiências e visões sobre a alimentação. É o espaço dos saberes, das reflexões acerca dos significados do alimento, do cotidiano de vida, da memória, da cultura em suas relações com o envelhecimento e com a saúde. É o campo que, a partir de Foucault ${ }^{4}$, é tomado para discussão das positividades dessas formações discursivas. Iniciativas que também têm trazido retorno para as práticas ambulatoriais e educativas em nutrição no interior do PNTI.

Assim, o enfoque das pesquisas dirige-se às condições de vida dos idosos, ao estado nutricional e ao consumo alimentar articulados à compreensão das práticas alimentares, da caracterização de atitudes, percepções, representações sobre alimentação, nutrição, saúde e doença no contexto das intensas trans- formações que vêm ocorrendo nos últimos séculos no campo da cultura alimentar.

Finalmente, o eixo da realização de práticas educativas e desenvolvimento de metodologias apropriadas ao trabalho com o público idoso que se apóia na perspectiva crítica da educação em saúde ${ }^{20,22}$. A partir desses referenciais, são privilegiados o respeito aos diferentes saberes e experiências, o diálogo, a interdisciplinaridade e a autonomia, considerando as possibilidades e limitações das condições de vida de cada um; é dada especial atenção às necessidades específicas do idoso, valorizando sua capacidade de aprender e criar e considerando as possíveis dificuldades inerentes ao processo de envelhecimento (limitações da capacidade auditiva, visual, de memória, concentração, entre outras); e são enfatizados os princípios do Sistema Único de Saúde, visando atender a toda a clientela sem discriminação (universalidade), respeitando as singularidades (eqüidade) em ações necessárias para a promoção, proteção e recuperação da saúde (integralidade). As atividades desenvolvidas sob o enfoque individual acontecem através da orientação nutricional personalizada. O olhar coletivo inclui os Grupos de Sala de Espera e o Grupo Roda da Saúde, atividades do Núcleo de Atenção ao Idoso da UnATI para discussão de temas relacionadas à saúde e envelhecimento; também é oferecido o Curso Nutrição e Terceira Idade, onde se procura não apenas transmitir informações, mas construir um espaço de reflexão e troca de conhecimentos e experiências cotidianas entre os profissionais, estudantes e os idosos, o que potencializa o pensar sobre a atenção ao idoso. 
Todo esse conjunto opera como um laboratório dinâmico no qual são experimentados diferentes procedimentos em consulta ambulatorial; temas e estratégias são discutidos; são produzidos materiais didáticos que possibilitam reflexões sobre as ações educativas, reconstruindo novas práticas voltadas tanto para os idosos quanto para os alunos que estão permanentemente presentes. Cabe registrar a necessidade da apresentação para a comunidade científica, para os serviços e para a sociedade dos modelos de práticas educativas aí implementados.

Após todos esses anos de atuação junto aos serviços de saúde e ao centro de convivência que compõem a UnATI, foram produzidos textos relativos a informação nutricional para idosos ${ }^{14,15}$, ao desenvolvimento de ações educativas na $\mathrm{UnATI}^{12}$ e a reflexões sobre alimentação, saúde e cultura na velhi$\mathrm{ce}^{13,18}$. A perspectiva de articulação entre ensino, pesquisa e extensão tem colocado a necessidade de avaliação da produtividade da equipe do PNTI em matéria de publicações relativas geração de conhecimentos e de saberes e de desenvolvimento de técnicas de atuação em serviços. Essa produção é considerada, por nós, ainda incipiente. Identificamos que o intenso envolvimento com as ações extensionistas e com as complexas ações interdisciplinares ocupam a maior parte do nosso tempo, em detrimento de investimentos em publicações. Estes aspectos apresentam-se como um desafio: como gerenciar a organização/desenvolvimento das atividades de forma a contemplar, com qualidade, a prática extensionista, a pesquisa e a geração de publicações?
Talvez seja necessária maior qualificação da equipe no campo da ciência para que esse diálogo tão desejado entre extensão e pesquisa possa se materializar através de publicações academicamente relevantes. Talvez seja necessário valorizar adequadamente a produção de conhecimentos e saberes provenientes da extensão, que trazem em seu bojo a riqueza das experiências acumuladas. Talvez seja preciso reorientar as prioridades buscando racionalizar o tempo disponível, suprimindo algumas atividades para incluir a publicação de trabalhos científicos. Talvez a estrutura de distribuição de cargas horárias docentes na UERJ possa acontecer de modo a considerar com mais profundidade avaliações de mérito dos projetos implementados e de seus potenciais possibilitando a incorporação de novos docentes em seus quadros. Talvez a constituição de políticas institucionais que valorizem, em algum grau, a realização de atividades articuladas de extensão, ensino e pesquisa com retornos concretos para os projetos e docentes envolvidos na consecução de propostas que se enquadrem no escopo dessas políticas. Talvez um pouco de cada uma dessas possibilidades.

O fato é que são identificadas algumas limitações importantes que exigem de nós novas posturas. Principalmente, a necessidade de escrever, de registrar o que é feito. Estão sendo gerados novos conhecimentos e saberes, novas técnicas, ainda encerradas nos muros da Universidade. Múltiplos fatores podem ser elencados na identificação da determinação desses limites. Certamente, ações individuais e institucionais precisam ser implementadas para a superação desses limitantes que, acreditamos, 
sejam característicos, em algum grau, das atividades extensionistas na UERJ e no Brasil.

\section{Referências}

1. Andrade LB, Silva EP. A reforma da universidade começa pela extensão. Interagir: pensando a extensão. 2003; 4:25-38.

2. Canguilhem G. O normal e o patológico. Rio de Janeiro: Forense-Universitária; 1982.

3. Castro LMC. A universidade, a extensão universitária e a produção de conhecimentos emancipadores: ainda existem utopias realistas [tese]. Rio de Janeiro: Universidade do Estado do Rio de Janeiro; 2004.

4. Foucault M. As palavras e as coisas: uma arqueologia das ciências humanas. São Paulo: Martins Fontes; 1990.

5. Goldenberg M. A arte de pesquisar: como fazer pesquisa em Ciências Sociais. Rio de Janeiro: Record; 1997.

6. Instituto Brasileiro de Geografia e Estatística. Censo demográfico 1991: resultados do universo: microdados. Rio de Janeiro: IBGE, 2002. 21 CD-ROM; IBGE, Censo

Demográfico 2000. tabela 13 Disponível em: URL: http:/ /www.ibge.gov.br

7. Machado R. Ciência e saber: a trajetória da arqueoleogia de Foucault. Rio de Janeiro: Edições Graal; 1988.

8. Maldonado LA, Paiva EV. A iniciação científica na graduação em Nutrição: possibilidades e contribuições para a formação profissional. In: Calazans J., organizador. Iniciação Científica: construindo o pensamento crítico. São Paulo: Cortez; 1999. p. 141-62.
9. Melo EM, et al. Promoção da saúde do adolescente: experiência de extensão, ensino e pesquisa do projeto morada nova. In: Anais do $2^{\circ}$ Congresso Brasileiro de Extensão Universitária; Belo Horizonte, Brasil; 2004.

10. Ministério da Educação. Diretrizes Curriculares Nacionais dos Cursos de Graduação em Enfermagem, Medicina e Nutrição. Diário Oficial da União de Mar. 10, 2001, Seção 1E, p. 131.

11. Nogueira ML. Extensão Universitária: diretrizes conceituais e políticas. Fórum PROEX/UFMG; Belo Horizonte, Brasil; 2000.

12. Pacheco LC, Menezes MFG. In: Assis M. Promoção da saúde e envelhecimento: orientações para o desenvolvimento de ações educativas com idosos. Rio de Janeiro: CRDE/UnATI/ UERJ; 2002. p. 42-56. Disponível em: URL: http:/ / www.unati.uerj.br

13. Prado SD, et al. Alimentação, saúde e cultura: algumas reflexões sobre uma experiência com narrativas de idosos. Textos Envelhecimento 2005; 8(3):361-79.

14. Prado SD, Tavares ED, Veggi AB. Nutrição e saúde no processo de envelhecimento. In. Veras RP. Terceira idade: desafios para o próximo milênio. Rio de Janeiro: RelumeDumará; 1999.

15. Prado SD, Tavares EL. Alimentação saudável na terceira idade. In. Assuero LS, Caldas CP. Saúde do idoso: a arte de cuidar. Rio de Janeiro. Interciência; 2004.

16. Rocha RMG. Produção do conhecimento e metodologia da extensão. In: Anais do I Congresso Brasileiro de Extensão Universitária; João Pessoa, Brasil; 2002.

17. Sant'Anna MJG. UnATI, a velhice que se aprende na escola: um perfil de seus usuários. In. Veras RP. Terceira idade: desafios para o próximo milênio. Rio de Janeiro: RelumeDumará; 1999. 
18. Santos DM, Menezes MFG. Representações da alimentação saudável em idosos: UERJ sem muros - 1999 a 2001. In: VII Congresso Brasileiro de Saúde Coletiva; Rio de Janeiro, Brasil; 2003.

19. Stengers I. Da racionalidade científica (capturas, eventos, interesses). In. Quem tem medo da ciência: ciências e poderes. São Paulo: Siciliano;1990. p. 77-109.

20. Stotz EN. Enfoques sobre educação e saúde. In. Valla VV, Stotz EN. Participação popular, educação e saúde: teoria e prática. Rio de Janeiro: Relume-Dumará; 1993.
21. Universidade do Estado do Rio de Janeiro. Sub-reitoria de Extensão e Cultura. Projetos, Programas e Produtos de Extensão.

Disponível em: URL: http:/ / www.sr3.uerj.br/depext.

22. Vasconcelos EM. A saúde nas palavras e nos gestos: reflexões da rede de educação popular em saúde. São Paulo: HUCITEC; 2001.

23. Veras RP. Saúde e envelhecimento. Cad. Saúde Pública 2003; 19(3):700-1. 